\title{
GCU
}

Glasgow Caledonian

University

University for the Common Good

\section{Interventions for reducing sedentary behaviour in community-dwelling older adults}

Chastin, Sebastien; Gardiner, Paul A.; Ashe, Maureen C.; Harvey, Juliet A.; Leask, Calum F.; Balogun, Saliu; Helbostad, Jorunn L.; Skelton, Dawn A.

Published in:

Cochrane Database of Systematic Reviews

DOI:

10.1002/14651858.CD012784

Publication date:

2017

Document Version

Publisher's PDF, also known as Version of record

Link to publication in ResearchOnline

Citation for published version (Harvard):

Chastin, S, Gardiner, PA, Ashe, MC, Harvey, JA, Leask, CF, Balogun, S, Helbostad, JL \& Skelton, DA 2017, 'Interventions for reducing sedentary behaviour in community-dwelling older adults', Cochrane Database of Systematic Reviews, no. 9. https://doi.org/10.1002/14651858.CD012784

\section{General rights}

Copyright and moral rights for the publications made accessible in the public portal are retained by the authors and/or other copyright owners and it is a condition of accessing publications that users recognise and abide by the legal requirements associated with these rights.

Take down policy

If you believe that this document breaches copyright please view our takedown policy at https://edshare.gcu.ac.uk/id/eprint/5179 for details of how to contact us. 


\section{(E) Cochrane Library}

Cochrane Database of Systematic Reviews

\section{Interventions for reducing sedentary behaviour in community-dwelling older adults (Protocol)}

Chastin S, Gardiner PA, Ashe MC, Harvey JA, Leask CF, Balogun S, Helbostad JL, Skelton DA

Chastin S, Gardiner PA, Ashe MC, Harvey JA, Leask CF, Balogun S, Helbostad JL, Skelton DA. Interventions for reducing sedentary behaviour in community-dwelling older adults.

Cochrane Database of Systematic Reviews 2017, Issue 9. Art. No.: CD012784.

DOI: 10.1002/14651858.CD012784.

www.cochranelibrary.com 
TABLE OF CONTENTS

HEADER . . . . . . . . . . . . . . . . . . . . . . . . . . . . . . . . . . . . 1

ABSTRACT . . . . . . . . . . . . . . . . . . . . . . . . . . . . . . . . . . . . . . . 1

BACKGROUND . . . . . . . . . . . . . . . . . . . . . . . . . . . . . . . . . . . .

OBJECTIVES . . . . . . . . . . . . . . . . . . . . . . . . . . . . . . . . . . . . . . .

METHODS . . . . . . . . . . . . . . . . . . . . . . . . . . . . . . . . . . . . . .

ACKNOWLEDGEMENTS . . . . . . . . . . . . . . . . . . . . . . . . . . . . . . . . . . . . . . . .

REFERENCES . . . . . . . . . . . . . . . . . . . . . . . . . . . . . . . . . . . . . . 7

APPENDICES . . . . . . . . . . . . . . . . . . . . . . . . . . . . . . . . . . . . . 9

CONTRIBUTIONS OF AUTHORS . . . . . . . . . . . . . . . . . . . . . . . . . . . . . . . . . . . . . . . . . . . .

DECLARATIONS OF INTEREST . . . . . . . . . . . . . . . . . . . . . . . . . . . . . . . . 11

SOURCES OF SUPPORT . . . . . . . . . . . . . . . . . . . . . . . . . . . . . . . . . . . . . . . . . .

Interventions for reducing sedentary behaviour in community-dwelling older adults (Protocol)

Copyright $\odot 2017$ The Cochrane Collaboration. Published by John Wiley \& Sons, Ltd. 


\title{
[Intervention Protocol]
}

\section{Interventions for reducing sedentary behaviour in community-dwelling older adults}

\author{
Sebastien Chastin ${ }^{1,2}$, Paul A Gardiner ${ }^{3,4}$, Maureen C Ashe ${ }^{5}$, Juliet A Harvey ${ }^{1}$, Calum F Leask ${ }^{1}$, Saliu Balogun ${ }^{6}$, Jorunn L Helbostad \\ 7, Dawn A Skelton ${ }^{1}$ \\ ${ }^{1}$ School of Health \& Life Sciences, Institute of Applied Health Research, Glasgow Caledonian University, Glasgow, UK. ${ }^{2}$ Department of \\ Movement and Sports Sciences, Ghent University, Gent, Belgium. ${ }^{3}$ Centre for Health Services Research, The University of Queensland, \\ Woolloongabba, Australia. ${ }^{4}$ Mater Research Institute, The University of Queensland, South Brisbane, Australia. ${ }^{5}$ Centre for Hip Health \\ and Mobility, Department of Family Practice, The University of British Columbia, Vancouver, Canada. ${ }^{6}$ Menzies Institute for Medical \\ Research, University of Tasmania, Hobart, Australia. ${ }^{7}$ Department of Neuroscience, Faculty of Medicine, Norwegian University of \\ Science and Technology, Trondheim, Norway
}

Contact address: Sebastien Chastin, School of Health \& Life Sciences, Institute of Applied Health Research, Glasgow Caledonian University, Cowcaddens Road, Glasgow, G4 0BA, UK. Sebastien.Chastin@gcal.ac.uk.

Editorial group: Cochrane Public Health Group.

Publication status and date: New, published in Issue 9, 2017.

Citation: Chastin S, Gardiner PA, Ashe MC, Harvey JA, Leask CF, Balogun S, Helbostad JL, Skelton DA. Interventions for reducing sedentary behaviour in community-dwelling older adults. Cochrane Database of Systematic Reviews 2017, Issue 9. Art. No.: CD012784. DOI: 10.1002/14651858.CD012784.

Copyright (C) 2017 The Cochrane Collaboration. Published by John Wiley \& Sons, Ltd.

\begin{abstract}
A B S T R A C T
This is a protocol for a Cochrane Review (Intervention). The objectives are as follows:

1. To identify the effects and assess the effectiveness of interventions to reduce sedentary behaviour (total sedentary time and the pattern of accumulation of sedentary time) in older adults.

2. To summarise the effects of interventions to reduce sedentary behaviour on quality of life, depression, and health status in older adults.
\end{abstract}

3. To summarise any evidence on the cost-effectiveness of interventions that reduce sedentary behaviour in older adults.

\section{B A C K G R O U N D}

\section{Description of the condition}

Sedentary behaviour has been defined as an activity where the predominant posture is sitting or lying and energy expenditure is low (Chastin 2013; SBRN 2012). Sedentary behaviour is dis- tinctly different from inactivity: inactivity is often regarded as not meeting physical activity guidelines for health, or too little exercise - sedentary behaviour is too much sitting (Owen 2010). So, standing for long periods would be considered inactivity, sitting or lying down (except sleep) for long periods would be sedentary behaviour. Going for a slow walk would not be considered being sedentary (as the person is not sitting) and if the person never or rarely gets warmer or out of breath (moderate activity) they would 
be categorised as being inactive. To reduce sedentary behaviour, we need to get people on their feet (if able) as often as possible in their waking hours but we are not necessarily asking them to perform activity to a level that is moderate or vigorous.

Evidence is increasing to suggest that sedentary behaviour, especially when accumulated in prolonged and continuous bouts, is detrimental to physical health (de Rezende 2014; Dunstan 2012; Henson 2013), mental health (Lauder 2006), quality of life (Laforge 1999), and bone health (Chastin 2014), in addition to being associated with all-cause mortality, disease incidence and hospitalisation (Biswas 2015). Prolonged screen-based sedentary activities, for example watching television, have been shown to be associated with depressive symptoms (Teychenne 2010), whilst metabolic syndrome has a significant correlation with lengthy sedentary periods (Bankoski 2011). Sedentary behaviour is also associated with lower odds of successful ageing (Dogra 2012). These deleterious health effects of sedentary behaviour are different to those of physical inactivity and largely independent of an individual's physical activity levels (Bankoski 2011). In fact, the latest meta-analysis reveals that over one hour of daily moderateintensity activity is required to attenuate the association between sedentary time and mortality (Ekelund 2016). Spending on average $80 \%$ of their time in a seated posture and with $67 \%$ being sedentary for more than 8.5 hours per day (Harvey 2013), older adults are the most sedentary segment of society and seldom engage in such high level of daily moderate-intensity activity.

The UK and other countries have now issued recommendations to reduce sedentary time as part of their physical activity guidelines (DoH 2011). These guidelines recommend reducing sedentary time in addition to engaging in moderate-intensity physical activity. The reasoning is that solely promoting physical activity does not necessarily reduce sitting time as one may achieve the recommended physical activity guidelines, yet still sit for large periods of the day (Dogra 2012; Katzmarzyk 2010). Indeed, the latest reviews indicate that interventions aimed at promoting physical activity are ineffective at reducing sedentary time (Martin 2015; Prince 2014). Experimental work indicates that time spent in moderateintensity activity tends to displace time that individuals usually spend in light activity and activities incidental to daily living but not sitting time (Gomersall 2015). Interventions devised specifically to reduce sedentary behaviour are needed and are the next step in this field of research (Owen 2011).

The aim of this review is to both synthesise and compare the current evidence on the effectiveness of interventions to reduce sedentary time amongst the older adult population.

\section{Description of the intervention}

This review will assess the effectiveness of interventions aimed specifically at reducing sedentary behaviour in communitydwelling older adults. Sedentary behaviour is ubiquitous and occurs throughout the day. In order to reduce sedentary time, in- terventions must specifically promote more time spent in upright postures throughout the day. Interventions can specifically focus on sedentary behaviour or be part of physical activity programmes which include a component specifically addressing sedentary behaviour (Martin 2015; Prince 2014). The type and components of interventions developed in adults (18 years and over) have varied widely and have been mapped against the taxonomy of behaviour change strategies (Gardner 2016; Michie 2013).

Some interventions target individuals on a one-to-one basis, or may look at a wider reach through communities; some provide feedback using monitors; and some just rely on general awareness raising. With advances in body-worn sensors and self-tracking technology the use of promoting devices is starting to appear in the literature. Finally some interventions are now considering environmental restructuring, such as standing desks and outdoor spaces.

Some of these interventions have been delivered via online media, for example using email messages (Adams 2013), whilst face-toface consultations have also been shown to provide fruitful outcomes in decreasing sedentary periods (Gardiner 2011). Modification of individual modalities, for example targeting cognitive behavioural therapy, has been the focus of numerous interventions (de Greef 2010).

The majority of interventions have focused on the individual, but others are attempting to modify more distal determinants of sedentary behaviour or even target a different level of determinants (Owen 2011). For example, environmental restructuring interventions modifying home and workplace layout or implementing outdoor spaces and facilities have all been acknowledged as potentially potent ways of reducing sedentary time (Gardner 2016; Tandon 2012).

Recent reviews of interventions in adults found that interventions that targeted sedentary behaviour specifically instead of physical activity were more successful in reducing sedentary time (Martin 2015; Prince 2014). A systematic review identified that interventions based on environmental restructuring, persuasion, or education were more successful in reducing sitting time and that selfmonitoring, problem solving, and restructuring the social or physical environment were particularly useful behaviour change techniques (Gardner 2016).

There are some limitations in interventions attempting to reduce sedentary time in older adults. Primarily, some sedentary activities older adults participate in, for example reading and socialising (Leask 2015), provide a mental health benefit (Alpass 2003), and facilitate cognitive function in ageing (Hertzog 2009). Therefore, although prolonged sedentary time may have a detrimental effect on physical health, some instances provide a positive mental health benefit and should not be decreased. Further activities, for example eating and resting, are essential for daily living and should not be altered. 


\section{How the intervention might work}

Interventions to reduce sedentary time in adults have been either interventions with a specific goal of increasing physical activity levels alongside reducing sedentary time or interventions aimed at reducing sedentary time only (Martin 2015; Prince 2014). Those focusing on reducing sedentary behaviour have resulted in a greater reduction of sedentary time (Martin 2015; Prince 2014). Indeed the determinants of sedentary behaviour are distinct from those of physical activity (Chastin 2015b), and the intervention must specifically address these determinants.

There are a number of different ways that sedentary behaviour interventions are predicted to decrease sedentary time or break up prolonged sitting time in older adults.

- Providing information: interventions could be used to educate individuals on the benefits of decreasing their overall sedentary time and breaking up prolonged sedentary periods, by using consultations/interviews, reviewing their own behaviour (self-monitoring by diary) or employing a feedback system. An example of such a feedback system would be the use of objective monitors to detect sedentary behaviour and print out feedback to be discussed with the individual to identify times when prolonged sitting could be reduced (i.e. by avoiding preferred seated activities such as social events, reading and knitting).

- Prompting: real-time behaviour prompts using wearable sensor and mobile technology that detect prolonged sedentary periods and prompt the individual to rise and move. Less frequent reminders by email and phone message may act as a less regular prompt system.

- Environmental restructuring: interventions may alter indoor or outdoor spaces to attempt to decrease individuals' sedentary time. More specifically, home or care setting layout changes might be considered, to encourage individuals to sit less. Standing desks and perching stools rather than comfortable seats are some potential examples of this.

- Policy change: organisations which provide services and care for older adults might change working practices to encourage individuals to sit less by, for example, using chair movement monitors, or encouraging staff to mobilise patients on a more regular basis.

\section{Why it is important to do this review}

The benefits of being physically active for older adults' health are well established (Kerr 2012; King 2001); and evidence suggesting the effectiveness of several intervention types to increase physical activity has been summarised in several review articles (King 1998; Van der Bij 2002). Despite these findings, older adults spend large periods of the day being sedentary, regardless of whether they are physically active (Harvey 2013; Harvey 2015). Increased sedentary time is associated with poorer health outcomes in older adults (de
Rezende 2014), including physical function (Rosenberg 2015), onset of frailty (Song 2015), and less successful ageing (Dogra 2012). There is now robust epidemiological evidence showing that the effect on health of prolonged sedentary time cannot be compensated for by adherence to physical activity and any exercise protocol (Biswas 2015). Consequently, interventions that specifically target sedentary behaviour have been advocated in this population in addition to promoting physical activity (Sparling 2015; Manns 2012). Older adults are potentially the population that might benefit the most from a reduction of sedentary time, because they are the most sedentary group and have the highest chronic disease burden (Harvey 2013; Harvey 2015). In addition, qualitative research reveals that older adults consider interventions to reduce sedentary behaviour more acceptable and desirable for them to adhere to than exercise programmes (Greenwood-Hickman 2015). To date, no reviews are available which have summarised study findings which aim to reduce or change sedentary patterns in older adults. Recently two reviews summarised the evidence in adults, including older adults (Martin 2015; Prince 2014). They included studies with a control or comparison group and identified four studies in older adults, but none with a sole focus on sedentary time. Compiling this information may help inform future interventions regarding the most successful and efficient methods to be found in the literature to decrease sedentary time in the population group with the largest amount and highest prevalence of sedentary time (Harvey 2015).

\section{O B J E C T I VES}

1. To identify the effects and assess the effectiveness of interventions to reduce sedentary behaviour (total sedentary time and the pattern of accumulation of sedentary time) in older adults.

2. To summarise the effects of interventions to reduce sedentary behaviour on quality of life, depression, and health status in older adults.

3. To summarise any evidence on the cost-effectiveness of interventions that reduce sedentary behaviour in older adults.

\section{METHODS}

\section{Criteria for considering studies for this review}

\section{Types of studies}

Due to the early nature of interventions to reduce sedentary time or change prolonged sitting patterns, this review will be not be 
restricted to randomised controlled trials. Despite the potential for bias in other study designs, the following study types will be included.

- Randomised controlled trials (RCTs).

- Cluster RCTs.

- Non-randomised controlled trials (NRCTs).

- Controlled before-after (CBA) studies with at least two intervention sites and two control sites.

- Cross-over studies.

- Interrupted Time Series (ITS) with a clearly defined point in time when the intervention occurred and at least three datapoints before and three after the intervention).

\section{Types of participants}

The inclusion criteria for data from studies will be as follows.

1. Adults aged 60 years and over.

2. Community-dwelling at home or living in a place of residence that does not provide nursing care, for example sheltered housing or residential complexes and not care or nursing homes.

3. Participants may have multiple co-morbidities or be disease free.

4. Studies investigating interventions in specific clinical populations only (e.g. stroke survivors) will be excluded.

\section{Types of interventions}

The review will include studies that report the following interventions (population-, community- or individual-based) designed to reduce sedentary time and particular sedentary behaviours, for example sitting time and watching television.

1. Interventions targeting the raising of awareness and provision of information. This might include interventions which use IT and social networks in addition to peer support networks.

2. Interventions which use prompting on multiple occasions (vibration monitors, phones, emails) to raise awareness and provide feedback on sedentary behaviour.

3. Interventions which attempt to alter the environment, for example modifying the layout of indoor and outdoor spaces and furniture.

4. Interventions that aim to change policy and practice in people's work with older people, for example motivating staff to encourage older people to move more frequently.

Interventions will be categorised as short term (up to 3 months in duration), medium term (3 months to 1 year in duration) or long term (longer than 1 year in duration).

The review will not consider interventions aimed solely at increasing physical activity; however interventions that target both an increase in physical activity and a reduction in sedentary behaviour will be included but presented separately.
We will compare the interventions described above with no intervention or with standard care or with other active interventions.

\section{Types of outcome measures}

\section{Primary outcomes}

1. Time spent sedentary (measured by self-reported measures or objective measures).

2. Time spent in specific sedentary behaviour (e.g. time spent watching TV) as defined by the International Classification of Sedentary Behaviours (Chastin 2013).

3. Pattern of accumulation of sedentary time (e.g. number of breaks in sedentary time (Chastin 2015a; Healy 2008), distribution of bouts of sedentary time (Chastin 2010)).

\section{Secondary outcomes}

1. Quality of life.

2. Depression.

3. Health status (improvement in physical function, cardiovascular and metabolic outcomes, and improvement in cognition).

4. Cost-effectiveness.

\section{Search methods for identification of studies}

The draft search strategy for MEDLINE will be used as the basis for search strategies in the other databases.

The search string is presented in Appendix 1.

\section{Electronic searches}

We will search all potentially eligible published studies. We will create a comprehensive search strategy, derived from terms related to sedentary behaviour, study design type, and population of study participants. Search criteria will be informed by reviews by Martin and colleagues and Prince and colleagues (Martin 2015; Prince 2014). We will search the following electronic databases to identify a list of potential articles that are written in English.

1. Cochrane Central Register of Controlled Trials (CENTRAL) in the Cochrane Library.

2. Embase (Embase.com).

3. MEDLINE (PubMed).

4. World Health Organization (WHO) International Clinical Trials Registry Platform (ICTRP) search portal (apps.who.int/ trialsearch).

5. CINAHL EBSCO (Cumulative Index to Nursing and Allied Health Literature).

6. ClinicalTrials.gov (ClinicalTrials.gov).

7. PsycINFO (ProQuest). 
8. PEDro (Physiotherapy Evidence Database;

www.pedro.org.au).

9. EPPI-Centre Databases (eppi.ioe.ac.uk/cms/Default.aspx?

tabid=185), e.g. Trials Register of Promoting Health

Interventions (TRoPHI).

10. AMED.

\section{Searching other resources}

We will consult the reference list of articles for additional studies. We will also contact experts in the field through the International Physical and Environment Network (www.ipenproject.org/ index.html), International Society for Physical Activity and Health (www.ispah.org), Sedentary Behaviour Research Network (www.sedentarybehaviour.org) and other leading international research networks in order to identify any additional work which is unpublished.

\section{Data collection and analysis}

\section{Selection of studies}

Two authors will review studies independently through three stages: 1) title screening; 2) abstract screening; 3) full-text screening. Authors will code the studies at each stage of the review process as 'included' or 'excluded'. If they encounter any inclusion or exclusion discrepancies, the two authors will either resolve them by discussion, or consult a third author who will act as arbitrator. We will exclude duplicate studies. We will record and present the inclusion process in a PRISMA flow diagram (Moher 2009).

\section{Data extraction and management}

We will extract the following data from all studies using the Cochrane Public Health extraction template.

1. Methodological information: study design, intervention duration, follow-up duration, study date, context.

2. Participant information: inclusion and exclusion criteria, sample size, age range, pre-intervention and post-intervention sitting time, health status, socioeconomic status.

3. Sedentary behaviour monitoring method.

4. Intervention information: intervention description, length and if a comparison intervention or control group was used.

5. Outcome information: reporting both primary and secondary outcomes.

6. Additional information: conflicts of interest; intervention fidelity (whether the intervention was delivered as planned); information on the nature and extent of any additional actions given as part of the intervention (co-interventions); intervention costs; source of study funding.
Two authors will independently extract data on each study. They will resolve any disagreements regarding data extraction by discussion or, if needs be, by consulting a third author. They will initially enter all extracted information into a spreadsheet before one author transfers it to Review Manager 5 (RevMan 5). A second author will double check the data once it is transferred to ensure correct data entry.

We will present these data in a 'Characteristics of included studies' table.

\section{Assessment of risk of bias in included studies}

Two review authors will independently assess the included studies with respect to risk of bias. We will assess RCTs using Cochrane's 'Risk of bias' tool (Higgins 2011). This tool assesses five domains: selection bias (sequence generation and allocation concealment); performance and detection bias (blinding); attrition bias (incomplete outcome data, withdrawals, dropouts, protocol deviations); reporting bias; and an open "other bias" category (such as baseline comparability for age, gender and occupation).

For the other study designs, we will use study-appropriate risk of bias domains as developed by the EPOC group (EPOC 2017): similar baseline characteristics, similar baseline outcome measures, reliable primary outcome measures and adequate protection against contamination. For ITS studies we will consider the following domains: was the intervention independent of other changes, was the shape of the intervention effect pre-specified, was the intervention unlikely to affect data collection, was knowledge of the allocated intervention adequately prevented during the study, were incomplete outcome data adequately addressed, was the study free from selective outcome reporting and was the study free from other risks of bias? For cluster RCT we will consider (i) recruitment bias; (ii) baseline imbalance; (iii) loss of clusters; (iv) incorrect analysis; and (v) comparability with individually randomised trials.

We will resolve any disagreement in the assessment between the two review authors by discussion or consensus with a third review author. We will assess the likely magnitude and direction of the bias on the findings.

We will assess each potential source of bias as high, low or unclear and provide a justification for our judgment in the 'Risk of bias' tables. We will not consider blinding of participants and personnel for risk of bias assessment as it is not possible to blind them in studies trying to modify sedentary behaviour. We will consider each blinding of outcome assessment differently when sedentary time is assessed objectively or by self-report. Studies will be deemed to have a high risk of bias if we judge the study to have a high risk of bias in any of the criteria (excluding blinding of participants and personnel).

\section{Measures of treatment effect}

In order to calculate treatment effects, we will enter outcome data from all included studies into RevMan 5. We will use risk ratio 
(RR) for dichotomous outcomes, and mean and mean differences (MDs) for continuous outcomes. We will use the generic inverse variance method when continuous outcomes are not available but only effect estimates (such as $95 \%$ confidence intervals or standard error).

\section{Unit of analysis issues}

If a selected study includes multiple trial arms, then we will include only the relevant arms. If trials with multiple relevant intervention arms incorporate a standard care or placebo or control arm to their trial, we will include them and compare each arm to the control. For cluster-randomized studies that report sufficient data to be included in the meta-analysis but do not correct for the design effect, we will calculate the design effect based on a fairly large assumed intracluster correlation coefficient of 0.10 .

If the study used a cluster design, we will assess whether the reported results were properly adjusted to account for clustering effects and what method was used.

\section{Dealing with missing data}

If there are missing numerical outcome data, we will contact study authors in an attempt to retrieve them. In the event that we are unable to gather these data then a risk of bias might be introduced, and we will conduct a sensitivity analysis to assess the impact of including the study. Two authors will then discuss whether the study should be included in the analysis or excluded due to a high potential risk of bias.

If outcome data such as standard deviations are missing and we cannot obtain them from the authors, we will derive them from other available statistics following the methods described in the Cochrane Handbook for Systematic Reviews of Interventions ( Higgins 2011).

\section{Assessment of heterogeneity}

We will class studies as homogeneous when the results are similar with regards to participants, interventions and outcomes.

We will analyse heterogeneity using the $\mathrm{I}^{2}$ and $\mathrm{Chi}^{2}$ statistic and corresponding P value, as well as $\mathrm{Tau}^{2}$ for random-effects metaanalysis. We will report degrees of heterogeneity as a low degree of heterogeneity ( $25 \%$ to $50 \%$ ), a moderate degree of heterogeneity $(50 \%$ to $75 \%)$ or a high degree of heterogeneity ( $75 \%$ or higher).

\section{Assessment of reporting biases}

Provided that we are able to pool more than 10 trials in any metaanalysis, publication bias will be assessed using the funnel plot and Egger's test methods (Egger 1997).

\section{Data synthesis}

We will sort the included studies according to the intervention, measures and comparisons made. We will assess results for each comparison and collate them separately. The findings of the studies will be systematically examined and integrated across studies. The included studies will be described and grouped by study design, population, outcomes, and setting. We will tabulate data and use them to explore relationships within and between included studies in a narrative summary.

If population, study design, intervention and outcome measures are similar enough across studies, we will conduct meta-analyses using RevMan 5. If outcomes within a comparison are obtained from several study designs, we will present results from non-randomised designs separately.

We will use RevMan 5 to gather homogeneous data (including economic data) from studies. We will perform meta-analysis if appropriate. We will use a fixed-effect model for homogeneous data, whilst if we find data to be statistically heterogeneous we will use a random-effects model. We will analyse intervention effects according to primary intervention categories: self-reported sitting time, television viewing, miscellaneous sedentary time, and objectively measured sitting time.

If we do not consider meta-analysis to be meaningful or appropriate, we will present descriptive data in text and tables and synthesise narratively in the main text of the review. We will structure the narrative synthesis according to study design or study quality and intervention setting.

\section{Quality of the evidence}

We will present the main outcome measures in a 'Summary of findings' table and will assess the quality of evidence for each of the outcomes using the GRADE methodology (Guyatt 2011). Using GRADE, we will reflect the extent to which we have confidence that the estimates of effect are correct. Our confidence will be presented as either high, moderate, low or very low. We will assess the results for each outcome measure against eight criteria. The first five criteria are considered for possible downgrading of the quality of documentation: study quality (risk of bias); consistency (consistency between studies); directness (the same study participants, intervention and outcome measures in included studies is for the people, measures and outcomes we wanted to study); precision of results; and reporting biases. Results can be upgraded by three criteria: strong or very strong associations between intervention and outcome; large or very large dose-response effects; and where all plausible confounders would have reduced the effect.

\section{Subgroup analysis and investigation of heterogeneity}

If there are sufficient data, we will explore subgroup analysis on the primary research objective. The following subgroups will be examined using the outcomes of self-reported sitting time, television viewing, miscellaneous sedentary time and objectively measured sitting time. 
1. Gender.

2. Geographical location (e.g. difference between urban and rural settings).

3. Socioeconomic status.

4. Health status.

\section{Sensitivity analysis}

We will assess the robustness of our conclusions by performing a sensitivity analysis in which we will exclude studies judged to have a high risk of bias from the meta-analysis on primary outcomes.
We may conduct further sensitivity analyses based on study characteristics that emerge during the review process.

\section{ACKNOW LEDGEMENTS}

The authors wish to acknowledge the editorial team of the Cochrane Public Health Group and the librarians at Glasgow Caledonian University who will create and execute the search strategies for the electronic databases. No funding was used to prepare this protocol.

\section{R E F E R E N C E S}

\section{Additional references}

\section{Adams 2013}

Adams MM, Davis PG, Gill DL. A hybrid online intervention for reducing sedentary behavior in obese women. Frontiers in Public Health 2013;1:45.

Alpass 2003

Alpass FM, Neville S. Loneliness, health and depression in older males. Aging \& Mental Health 2003;7(3):212-6.

Bankoski 2011

Bankoski A, Harris TB, McClain JJ, Brychta RJ, Caserotti P, Chen KY, et al. Sedentary activity associated with metabolic syndrome independent of physical activity. Diabetes Care 2011;34(2):497-503.

\section{Biswas 2015}

Biswas A, Oh PI, Faulkner GE, Bajaj RR, Silver MA, Mitchell MS, et al. Sedentary time and its association with risk for disease incidence, mortality, and hospitalization in adults: a systematic review and meta-analysis. Annals of Internal Medicine 2015;162(2):123-32.

\section{Chastin 2010}

Chastin SFM, Granat M. Methods for objective measure, quantification and analysis of sedentary behaviour and inactivity. Gait \& Posture 2010;31(1):82-6.

Chastin 2013

Chastin S, Scwartz U, Skelton D. Development of a consensus taxonomy of sedentary behaviors (SIT): Report of Delphi Round 1. PLoS ONE 2013;8(12):e82313.

\section{Chastin 2014}

Chastin SFM, Mandrichenko O, Helbostadt JL, Skelton DA. Associations between objectively-measured sedentary behaviour and physical activity with bone mineral density in adults and older adults, the NHANES study. Bone 2014; 64:254-62.

Chastin 2015a

Chastin SFM, EgertonT, Leask C, Stamatakis E. Metaanalysis of the relationship between breaks in sedentary behavior and cardiometabolic health. Obesity 2015;23(9): 1800-10.

\section{Chastin 2015b}

Chastin SFM, Buck C, Freiberger E, Murphy M, Brug J, Cardon G, et al. Systematic literature review of determinants of sedentary behaviour in older adults: a DEDIPAC study. International Journal of Behavioral Nutrition and Physical Activity 2015;12(1):127.

\section{de Greef 2010}

de Greef K, Deforche B, Tudor-Locke C, de Bourdeaudhuij I. A cognitive-behavioural pedometer-based group intervention on physical activity and sedentary behaviour in individuals with type 2 diabetes. Health Education Research 2010;25(5):724-36.

\section{de Rezende 2014}

de Rezende LFM, Rey-López JP, Matsudo VKR, do Carmo Luiz, O. Sedentary behavior and health outcomes among older adults: a systematic review. BMC Public Health 2014; 14(1):333.

\section{Dogra 2012}

Dogra S, Stathokostas L. Sedentary behavior and physical activity are independent predictors of successful aging in middle-aged and older adults. Journal of Aging Research 2012;2012:Article ID 190654.

\section{DoH 2011}

Department of Health. Start active, stay active: a report on physical activity from the four home countries' Chief Medical Officers. www.gov.uk/government/ uploads/system/uploads/attachment_data/file/216370/dh_ 128210.pdf (accessed prior to 4 July 2017).

\section{Dunstan 2012}

Dunstan DW, Kingwell BA, Larsen R, Healy GN, CerinE, Hamilton MT, et al. Breaking up prolonged sitting reduces postprandial glucose and insulin responses. Diabetes Care 2012;35(5):976-83.

\section{Egger 1997}

Egger M, Davey Smith G, Schneider M, Minder C. Bias in meta-analysis detected by a simple, graphical test. British Medical Journal 1997;315:629-34. 


\section{Ekelund 2016}

Ekelund U, Steene-Johannessen J, Brown JW, Fagerland MW, Owen N, Powell KE, et al. Does physical activity attenuate, or even eliminate, the detrimental association of sitting time with mortality? A harmonised meta-analysis of data from more than 1 million men and women. Lancet 2016;388(10051):1302-10.

EPOC 2017

Cochrane Effective Practice, Organisation of Care (EPOC). Suggested risk of bias criteria for EPOC reviews. EPOC Resources for review authors. 2017; Vol. Available at: http://epoc.cochrane.org/epoc-specific-resources-reviewauthors.

\section{Gardiner 2011}

Gardiner PA, Eakin EG, Healy GN, Owen N. Feasibility of reducing older adults' sedentary time. American Journal of Preventive Medicine 2011;41(2):174-7.

\section{Gardner 2016}

Gardner B, Smith L, Lorencatto F, Hamer M, Biddle SJ. How to reduce sitting time? A review of behaviour change strategies used in sedentary behaviour reduction interventions among adults. Health Psychology Review 2016; 10(1):89-112.

Gomersall 2015

Gomersall SR, Maher C, English CK, Rowland A, Olds T. Time regained: when people stop a physical activity program, how does their time use change? A randomised controlled trial. PLoS ONE 2015;10(5):e0126665.

Greenwood-Hickman 2015

Greenwood-Hickman MA, Renz A, Rosenberg DE. Motivators and barriers to reducing sedentary behavior among overweight and obese older adults. Gerontologist 2016; Vol. 56, issue 4:660-8.

\section{Guyatt 2011}

Guyatt GH, Oxman AD, Schünemann HJ, Tugwell P, Knottnerus A. GRADE guidelines: a new series of articles in the Journal of Clinical Epidemiology. Journal of Clinical Epidemiology 2011;64(4):380-2.

\section{Harvey 2013}

Harvey JA, Chastin SFM, Skelton DA. Prevalence of sedentary behavior in older adults: a systematic review. International Journal of Environmental Research and Public Health 2013;10(12):6645-61.

Harvey 2015

Harvey JA, Chastin SF, Skelton DA. How sedentary are older people? A systematic review of the amount of sedentary behavior. Journal of Aging and Physical Activity 2015;23(3):471-87.

Healy 2008

Healy GN, Dunstan DW, Salmon J, Cerin E, Shaw JE, Zimmet PZ, et al. Breaks in sedentary time: beneficial associations with metabolic risk. Diabetes Care 2008;31(4): 661-6.

Henson 2013

Henson J, Yates T, Biddle SJH, Edwardson CL, Khunti K, Wilmot EG, et al. Associations of objectively measured sedentary behaviour and physical activity with markers of cardiometabolic health. Diabetologia 2013;56(5):1012-20.

Hertzog 2009

Hertzog C, Kramer AF, Wilson RS, Lindenberger U.

Enrichment effects on adult cognitive development. Psychological Science in the Public Interest 2009;9:1-65.

\section{Higgins 2011}

Higgins JP, Green S, (editors). Cochrane Handbook for Systematic Reviews of Interventions Version 5.1.0 (updated March 2011). The Cochrane Collaboration, 2011. Available from handbook.cochrane.org.

\section{Katzmarzyk 2010}

Katzmarzyk PT. Physical activity, sedentary behavior, and health: paradigm paralysis or paradigm shift?. Diabetes, 2010;59(11):2717-25.

\section{Kerr 2012}

Kerr J, Rosenberg DE, Nathan A, Millstein R, Carlson J, Crist K, et al. Applying the ecological model of behavior change to a physical activity trial in retirement communities: description of the study protocol. Contemporary Clinical Trials 2012;33(6):1180-8.

\section{King 1998}

King AC, Rejeski WJ, Buchner DM. Physical activity interventions targeting older adults. A critical review and recommendations. American Journal of Preventive Medicine 1998;15(4):316-33.

\section{King 2001}

King AC. Interventions to promote physical activity by older adults. Journals of Gerontology. Series A, Biological Sciences and Medical Sciences 2001;56:36-46.

\section{Laforge 1999}

Laforge RG, Rossi JS, Prochaska JO, Velicer WF, Levesque $\mathrm{D}, \mathrm{McH}$ orney C. Stage of regular exercise and health-related quality of life. Preventive Medicine 1999;28(4):349-60.

\section{Lauder 2006}

Lauder W, Mummery K, Jones M, Caperchione C. A comparison of health behaviours in lonely and non-lonely populations. Psychology, Health \& Medicine 2006;11(2): 233-45.

\section{Leask 2015}

Leask C, Harvey JA, Skelton DA, Chastin SFM. The context of sedentary behaviour in older adults (what, where, why, when and with whom). European Review of Aging and Physical Activity 2015;12:4-8.

\section{Manns 2012}

Manns PJ, Dunstan DW, Owen N, Healy GN. Addressing the nonexercise part of the activity continuum: a more realistic and achievable approach to activity programming for adults With mobility disability?. Physical Therapy 2012; 92(4):614-25.

\section{Martin 2015}

Martin A, Fitzsimons C, Jepson R, Saunders DH, van der Ploeg HP, Teixeira PJ, et al. EuroFIT consortium. Interventions with potential to reduce sedentary time in 
adults: systematic review and meta-analysis. British Journal of Sports Medicine 2015;49(16):1056-63.

\section{Michie 2013}

Michie S, Richardson M, Johnston M, Abraham C, Francis J, Hardeman W, et al. The behavior change technique taxonomy (v1) of 93 hierarchically clustered techniques: building an international consensus for the reporting of behavior change interventions. Annals of Behavioral Medicine 2013;46(1):81-95.

Moher 2009

Moher D, Liberati A, Tetzlaff J, Altman DG. Preferred reporting items for systematic reviews and meta-analyses: the PRISMA statement. PLoS Medicine 2009;6(7):b2535.

Owen 2010

Owen N, Healy GN, Matthews CE, Dunstan DW. Too much sitting: the population-health science of sedentary behavior. Exercise and Sport Sciences Reviews 2010;38(3): 105-13.

Owen 2011

Owen N, Sugiyama T, Eakin EE, Gardiner PA, Tremblay MS, Sallis JF. Adults' sedentary behavior determinants and interventions. American Journal of Preventive Medicine 2011;41(2):189-96.

Prince 2014

Prince SA, Saunders TJ, Gresty K, Reid RD. A comparison of the effectiveness of physical activity and sedentary behaviour interventions in reducing sedentary time in adults: a systematic review and meta-analysis of controlled trials. Obesity Reviews 2014;15(11):905-19.

\section{Rosenberg 2015}

Rosenberg DE, Bellettiere J, Gardiner PA, Villarreal VN, Crist K, Kerr J. Independent associations between sedentary behaviors and mental, cognitive, physical, and functional health among older adults in retirement communities.
Journals of Gerontology. Series A, Biological Sciences and Medical Sciences 2016;71(1):78-83. [10.1093/gerona/ glv103]

\section{SBRN 2012}

Sedentary Behaviour Research Network. Standardized use of the terms "sedentary" and "sedentary behaviours " Letter to the Editor. Applied Physiology Nutrition and Metabolism (joint publication with Movement and Sport Sciences) 2012;37:540-2.

\section{Song 2015}

Song J, Lindquist LA, Chang RW, Semanik PA, EhrlichJones LS, Lee J, et al. Sedentary behavior as a risk factor for physical frailty independent of moderate activity: Results from the Osteoarthritis Initiative. American Journal of Public Health 2015;105(7):1439-45.

Sparling 2015 Sparling PB, Howard BJ, Dunstan DW, Owen N. Recommendations for physical activity in older adults. British Medical Journal 2015;350:h100.

Tandon 2012

Tandon PS, Zhou C, Sallis JF, Cain K L, Frank LD, Saelens BE. Home environment relationships with children's physical activity, sedentary time, and screen time by socioeconomic status. International Journal of Behavioral Nutrition and Physical Activity 2012;9(1):88.

Teychenne 2010

Teychenne M, Ball K, Salmon J. Sedentary behavior and depression among adults: A review. International Journal of Behavioral Medicine 2010;17(4):246-54.

Van der Bij 2002

Van der Bij AK, Laurant MGH, Wensing M. Effectiveness of Physical Activity: A Review. American Journal of Preventive Medicine 2002;22(2):120-33.

* Indicates the major publication for the study

\section{A P P E N D I C ES}

\section{Appendix I. Draft search terms for PubMed}

Draft MEDLINE (Pubmed) search strategy terms. Sedentary terms, Study design terms and Population terms will be linked by a AND boolean operator

In addition filter will be applied to limit the search to human research and texts in English.

Sedentary terms

1. Sedentary lifestyle[MeSH Terms]

2. (((sedentary behavior[tiab]) OR sedentary behaviour[tiab]) OR sedentary lifestyle[tiab])

3. prolonged sitting[tiab]

4. (sitting time[tiab]) OR lying time[tiab]

5. computer[MeSH Terms]

6. ((computer use[tiab]) OR computer usage[tiab]) OR computer time[tiab]

Interventions for reducing sedentary behaviour in community-dwelling older adults (Protocol)

Copyright @ 2017 The Cochrane Collaboration. Published by John Wiley \& Sons, Ltd. 
7. ((internet use[tiab]) OR internet usage[tiab]) OR internet time[tiab]

8. television[MeSH Terms]

9. ((television viewing[tiab]) OR television watching[tiab]) OR television time[tiab]

10. ((TV viewing[tiab]) OR TV watching[tiab]) OR TV time[tiab]

11. (watch television[tiab]) OR view television[tiab]

12. (watch TV[tiab]) OR view TV[tiab]

13. ((screen watching[tiab]) OR screen use[tiab]) OR screen time[tiab]

14. ((screen entertainment[tiab]) OR screen behaviour[tiab]) OR screen behavior[tiab]

15. ((screen-based entertainment[tiab]) OR screen-based behaviour[tiab]) OR screen-based behavior[tiab]

16. reading time[tiab]

17. (games, video[MeSH Terms]) OR games, video[MeSH Terms]

18. (((computer game time[tiab]) OR computer gaming time[tiab]) OR video game time[tiab]) OR video gaming time[tiab]

19. (computer game[tiab]) OR computer gaming[tiab]

20. (video game[tiab]) OR video gaming[tiab]

21. automobile driving[MeSH Terms]

22. transport time[tiab]

AND

Study design terms

23. program evaluation[MeSH Terms]

24. intervention[tiab]

25. evaluation[tiab]

26. (randomised[tiab]) OR randomised[tiab]

27. (quasi-randomised[tiab]) OR quasi-randomized[tiab]

28. (non-randomised[tiab]) OR non-randomized[tiab]

29. randomised controlled trial[pt]

30. controlled clinical trial [pt]

31. randomly[tiab]

32. controlled clinical trial[pt]

33. alloc*[tiab]

34. trial[tiab]

35. group[tiab]

36. controlled trial[tiab]

37. quasi-experiment[tiab]

38. feasibility[tiab]

39. health promotion[tiab]

40. Health education[tiab]

41. (((behaviour therapy[tiab]) OR behavior therapy[tiab]) OR behavioural therapy[tiab]) OR behavioral therapy[tiab]

42. environmental design[tiab]

43. environmental restructur*[tiab]

AND

Population terms

44. aged[MeSH Terms]

45. middle aged[MeSH Terms]

46. senior*[tiab]

47. older adult*[tiab]

48. elderly[tiab]

49. older people[tiab]

50. (geriatric[Title/Abstract]) OR geriatrics[Title/Abstract]

Interventions for reducing sedentary behaviour in community-dwelling older adults (Protocol) 


\section{CONTRIBUTIONSOFAUTHORS}

Chastin, Skelton, Ashe, Helbostad, Gardiner and Leask conceived and developed the protocol. Harvey and Balogun commented on drafts of the protocol and search strategies. Gardiner and Leask will review all selected publications, with Skelton as adjudicator. Chastin will perform statistical analysis. Ashe, Helbostad, Harvey, Gardiner and Balogun will comment on drafts of the review.

\section{DECLARATIONSOF INTEREST}

Skelton, Chastin, Ashe, Helbostad, Gardiner, Harvey, Balogun and Leask: none known.

\section{SOURCES OF SUPPORT}

\section{Internal sources}

- New Source of support, Other.

\section{External sources}

- No sources of support supplied 